\title{
Editorial
}

\section{New Technologies: How Can Journals Best Serve Their Readers?}

\author{
Susan Springthorpe, BSc, MSc
}

The war against microbial contamination is neverending. Nosocomial infections are an undeniable fact, even in the best-run facilities. With ever-increasing populations, and more and larger institutions, the risk of acquiring infections rises. Furthermore, as the number of patients immunocompromised by age, disease, or medication increases, so does the risk that acquired infections might result in severe disease and a poor or fatal outcome. At the same time, the array of medical-device designs, and of the materials used in them, suggests that the field of device reprocessing is becoming a highly specialized one-and that is even without entering into the debate over reprocessing of single-use devices or understanding how each type of medical device deteriorates with use and age.

Infection control and central service personnel can become frustrated and battle-weary due to increased pressures on their time and facilities. New technologies, processes, and equipment that will assist them in their tasks are eagerly awaited, and this journal is an ideal vehicle for communicating such innovations to the healthcare community. This is particularly true for sterilization techniques.

All sterilization processes have their limitations, and it is only by understanding these process limitations and failure conditions that successful sterilization can be achieved on a routine and predictable basis. Even proven sterilization technologies, such as moist heat, require great attention to process validation and can fail for a variety of reasons.

Understandably, many studies of new technologies are funded by, and carried out with some direction from, the manufacturer(s) of the products, processes, or equipment. When successful, the results of these studies often are published in peer-reviewed journals. If the studies are carried out only in accord with the manufacturer's recommended use conditions, which presumably already have been deter- mined to give a successful result, then the manuscript amounts to a third-party endorsement, in a peer-reviewed journal, of a set of use conditions. This has the advantages of informing the healthcare community that the process exists and demonstrating to regulatory authorities that performance under the use conditions has been reviewed by third parties and published in a peer-reviewed journal. Beyond this, however, it tells the reader little that could not have been learned from the manufacturer's labeling instructions.

Could readers be better informed? Yes, they could. A critical analysis of relevance to practice and a detailed study that points in an obvious fashion to the variables likely to lead to failure conditions or potential harm to the patient would serve the infection control community much better. Would the manufacturer support or submit manuscripts that included failure conditions? Would the regulatory agencies accept publications that demonstrated success at the manufacturer's recommended use conditions but also showed conditions under which the product could fail? Would the reader be able to distinguish the value of the product or process when different conditions and varying outcomes are presented? This is a complex topic, but one that I believe deserves an airing, because proper performance of decontamination and sterilization procedures can save lives and reduce morbidity.

Of the articles in the current issue, at least two $0^{1,2}$ point to operational flaws in the use of established or new technologies. Rutala et al $^{1}$ describe burns received by patients from flash-sterilized instruments. It is impossible to know how many times this may have happened at different institutions, given the widespread use of flash sterilization. Would a published warning have prevented such events? Possibly, but not necessarily; such a warning would, however, be a prudent inclusion. 
The article by Penna et $\mathrm{a}^{2}$ looks at the presterilization microbial load (bioburden) on used medical devices after cleaning but before sterilization, as well as at the effectiveness of a gas plasma system at reducing the numbers of Bacillus subtilis spores inoculated onto such devices. As a result of their studies, the authors identified some critical points at which the sterilization process could fail. They demonstrated how the learning process helped them to obtain better presterilization counts through improved cleaning and to understand the importance of drying in controlling the presterilization bioburden. Mesophilic (and even occasionally thermophilic) spore-forming bacteria, as well as fungi, sometimes were found in relatively high numbers.

Sterilization of dried $B$ subtilis inocula also provided lessons for Penna et al. ${ }^{2}$ Condensation that formed in refrigerated items was found to inhibit the sterilization process, and a failure to ensure clear access of the plasma through narrow-lumen needles also led to variable results. Furthermore, the exact configuration and assembly of the items materially influenced access of the sterilizing plasma. The authors also pointed to the need for suitable clean sources of cleaning water and drying air.

Other than independent studies such as this one and those performed by Alfa and her colleagues (as cited by Penna et $\mathrm{a}^{2}$ ), what has been published about failure conditions for plasma sterilizers? Has any such published information come from the sterilizer manufacturers? With many such plasma units already in operation, are the staff who operate them really aware of the critical issues for their operation?

In this context, it is worth pointing to another paper in this issue. Rutala et $\mathrm{al}^{3}$ undertook a straightforward study of a new plasma sterilizer operating under the manufacturer's recommended conditions, with the conclusion that the equipment was able to sterilize highly contaminated lumened objects. While this may be perfectly accurate when field conditions replicate those of the experimental study, it is certainly worth making a careful comparison between this paper and the one by Penna et $\mathrm{al}^{2}$ in which actual instruments were used. Although these are similar technologies, they differ in the size of the equipment and the cycle time. Since the cycle time is shorter for the new technology, how is this likely to affect sterilization?

Would errors during reprocessing and sterilization in the field be reduced if articles evaluating new technologies evaluated critical process parameters that could compromise the process if not met and that potentially could lead to negative outcomes for the patient? This is by no means certain, because of human failings, but nonetheless the establishment of optimum modes of practice is dependent on having all the facts available. Furthermore, I firmly believe that people are more likely to be rigorous about routine practices if they understand their underlying rationales.

What therefore are the responsibilities of the various parties involved? Since patient health is at stake, analyzing and communicating critical control points that can make a potentially successful sterilization process fail should be done proactively rather than retrospectively. Introduction of a new technology should not be accepted without such an analysis. All parties have a role to play. The manufacturer should be able to identify any known or potential factors that might compromise sterilization. The scientist undertaking studies of a new technology should investigate the limits of a successful process. Journal editors, manuscript reviewers, and even the reader should ask appropriate questions when only successful conditions are reported.

While the peer-review process is usually the best way to examine the appropriateness and value of any particular contribution to the published literature, a reviewer can really only examine the material presented in a study and is unable to answer the "what if " questions of alternate study designs or conditions. It is really unfortunate, and potentially hazardous, if these independent evaluations of new technology do not challenge the systems being evaluated and if apparent flaws or critical parameters only surface after the technology has been in place for some time. It is extremely important for the infection control community to understand the ruggedness or fragility of the sterilization technology. Because later independent studies may be difficult to fund, it should be considered routine to address such issues in the initial independently conducted, manufacturersponsored process evaluations.

\section{REFERENCES}

1. Rutala WA, Weber DJ, Chappell KJ. Patient injury from flash-sterilized instruments. Infect Control Hosp Epidemiol 1999;20:458.

2. Penna TCV, Ferraz CAM, Cassola MA. The presterilization microbial load on used medical devices and the effectiveness of hydrogen peroxide gas plasma against Bacillus subtilis spores. Infect Control Hosp Epidemiol 1999;20:465-472.

3. Rutala WA, Gergen MF, Weber DJ. Sporocidal activity of a new low-temperature sterilization technology: the Sterrad 50 sterilizer. Infect Control Hosp Epidemiol 1999;20:514-516. 